\title{
How Crypto-Economics is Redefining Telemedicine
}

\author{
Brennan Bennett
}

With the proliferation of crypto-economics powered by mainstream adoption of blockchain technology, the core definition of telemedicine is poised to evolve.

Telemedicine was initially designed to address physical distance as a barrier to entry between patient and provider for health assessment and treatment. As the information age evolved, featured enhancements to telemedicine came along, such as widespread access to broadband for video conferencing and digitization of medical records. These advancements made it easier for both the patient and provider to communicate treatment plan progress or lack thereof.

As the information age brought an unprecedented evolution in communication, artificial intelligence (AI) brought an unprecedented evolution in trend analysis capabilities. Throughout the last two decades, Al has advanced to become a commodity in terms producing detailed trend analysis, which enables a reduction of resources for telemedicine practitioners whilst increasing diagnostic efficiency.

Enter blockchain - a technology born 100\% from within the digital era and designed from the ground up for a digital civilization. One thing blockchain technology is exceptionally good at is cutting out the middle man in every pay for service imaginable. In the healthcare space, blockchain entrepreneurs want to push beyond the meet-andgreet video functionality of telemedicine, beyond any sort of SaaS (Software as a Service) quasi Al solution that you may login to at any time and directly connect with one's health record.

The marriage of Al with blockchain's computational power has drawn a clear line in the sand. It is redefining what it means to be on the frontlines of precision science. Blockchain startups continue to push that line forward with innovative use of crypto- 
economics. Even more so by attaching crypto-economics to physical in-home, not evasive biomedical tests, as well as a host of personalized biomedical products, essentially setting the stage to eliminate the need for telemedicine to have a human on the other end of the line. With the philosophy of telemedicine in mind, one can be certain that in-house precision medicine tests and personalized biomedical products powered by decentralized ecosystems that run on crypto-economics encompass are all going to work together to generate the next evolution of HIT (healthcare information technology) innovation.

\section{Personalized OTC Skincare}

One such example test is an in-house skin care examination that tailors skin care regiments to an individual's unique biological makeup. The in-home, non-invasive epigenetic skin care test reveals the dynamic state of the person's skin, enabling the personalization of skin care products and regimens

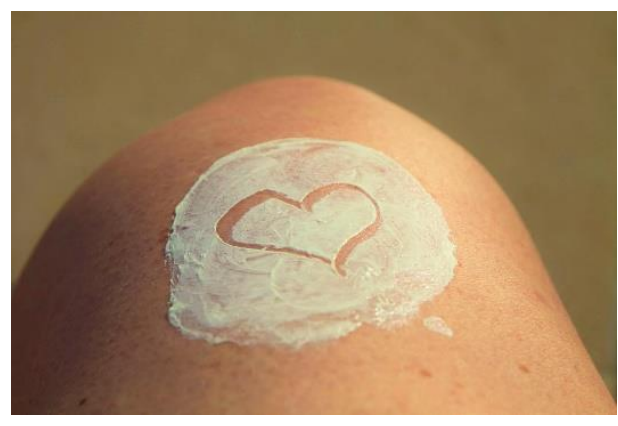
that best fits each participant's biological needs. Once the sample is received, the participant's unique biochemistry is analyzed to identify key variables affecting the participant's skin and personalizing recommendations to improve skin vitality.

When asked about the impact of blockchain technology in HIT space, EpigenCare cofounder William Lee said, "The blockchain facilitates a third party, decentralized means to anonymously link consumer data with commercial motives without sacrificing privacy while simultaneously allowing incentive models to be built through tokenization of data. This sort of link is a disruptive technology because consumers are more inclined to permit commercial entities to use their data and offer better solutions to them."

Targeted personalized solutions to the consumer, while still ensuring participant privacy, will create an anonymous link between the company purchasing of the data and the customer who falls within that data segment. 
"Over the next few years, we will see more for-profit companies in the healthcare space leverage blockchain technology to mitigate the privacy barrier to data-driven business models. EpigenCare intends to pioneer this movement" says Lee.

Decentralized is ultimately planning on leveraging crypto economics to create a supply and demand model for biomedical data. On the supply side, there are the tangible epigenetic data profiles generated from the consumer. On the demand side, skincare companies will want to leverage epigenetic data for research.

\section{Personalized Wellness}

In a nod to its telemedicine roots, blockchain HIT innovators are developing ecosystems to incentivize anonymous health data security via sharing to trusted parties. Anonymous Health Tokens (AHT), for example, are a type of token that can be acquired by participants as a reward for sharing anonymous health data. When used on their respective ecosystem, AHT helps monitor wellness by allowing the participant to store health data on the blockchain powered data ecosystem.

A comparison of blockchain vs. telemedicine features is listed in Table 1.

Table 1. Blockchain vs. telemedicine features

\section{Blockchain \\ Telemedicine}

Test for nutrient and hormone imbalances Talk about a test, then order it in real time

Dispense medicines based on the Get a prescription to order medicine patient's unique needs $100 \%$ control of medical and health No control of medical and health history history

\section{Optimize Your Biology}


Deep learning and natural language processing are now being integrated into blockchain innovation to remove not only the paperwork patient interactions, but the need to enter personal information at the medical appointment. Artificial intelligencepowered biology healthcare platforms can generate insights on blood, genomics, microbiome, environmental, and anatomical data.

The NEURON (NRN) token is a rewarded for participating and sharing data garnered from interacting with a deep learning Al interface with specific medical verticals. Naming the token NEURON is, of course, a throwback to the electrical pathways of the human body, of which there are an estimated 86 billion, which, in good measure, is the number of tokens the crypto economy will run on. Once fully operational, the token economics of the NEURON network will enable participants to use their health record as a financial asset (i.e., sell their data for research), knowledge asset (Al will notify participant of related medical advancements), and ecosystem asset (one's investment in health is helping others).

\section{What to Do With (Crypto) Currency}

While it may be exciting to participate in the next generation of HIT, it's hard to say when crypto-economics will be stable enough to earn substantial income by simply participating in various blockchain ecosystems. It is important to say however, that this time is relatively near. As of this writing, there are nearly 45 crypto tokens solely devoted to healthcare in some way shape of form, including a token for healthy pets.

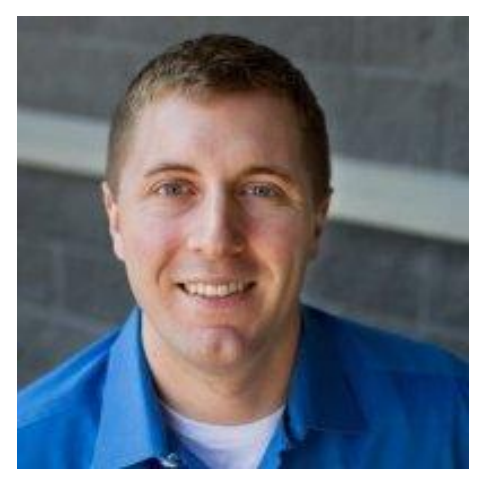

Brennan Bennett is the Founder and Managing Editor of Blockchain Healthcare Review, a publication covering technical innovations in blockchain technology and how it is 
reshaping the future of health data governance. He is an experienced HIT strategy consultant with a background in the Fortune 100 space. Additionally, Brennan holds a M.S. in Biomedical Informatics from Rutgers-The State University of New Jersey.

This is an open access article distributed in accordance with the Creative Commons Attribution Non-Commercial (CC BY-NC 4.0) license, which permits others to distribute, adapt, enhance this work non-commercially, and license their derivative works on different terms, provided the original work is properly cited as first published in TeleHealth and Medicine Today, and the use is non-commercial. See:

http://creativecommons.org/licenses/by-nc/4.0.

No endorsement of any product(s) mentioned in this article is to be inferred, nor is it implied by Telehealth and Medicine Today or its publisher, Partners in Digital Health.

Department: Blockchain

Tags: artificial intelligence, blockchain technology, crypto-economics, telemedicine, telehealth 